\title{
A NEW ALGORITHM FOR COMPUTING LIQUID CRYSTAL STABLE CONFIGURATIONS: THE HARMONIC MAPPING CASE*
}

\author{
FRANÇOIS ALOUGES ${ }^{\dagger}$
}

\begin{abstract}
In this article, we propose a new algorithm for minimizing the energy of a nematic liquid crystal. Based on the equal elastic constants Oseen-Frank model, the problem reduces to finding harmonic minimizing maps that take values into the unit sphere of $\mathbb{R}^{3}$. The convergence of this algorithm is proved in a continuous setting. Then, numerous numerical results that show its efficiency are given.
\end{abstract}

Key words. nematic liquid crystals, harmonic maps, nonconvex optimization, finite differences, relaxation method, Uzawa method, conjugate gradient method

AMS subject classifications. 35A40, 65C20, 65N30

PII. S0036142994264249

1. Introduction. In recent years, liquid crystals have been of constant interest for mathematicians and physicists. Several models were proposed (harmonic maps, Oseen-Frank model, Ericksen model) to explain the defects in the structure of nematics. Working with the Oseen-Frank model, nematic liquid crystals are naturally represented by an $S^{2}$-valued map, with which one associates an energy that depends on some elastic constants (see formula (2)). For some values of these constants the energy reduces to the Dirichlet energy (5), and the stable configurations of the liquid crystal are closely related to minimizing harmonic $S^{2}$-valued maps. Even if it appears to be quite restrictive, this case is nonetheless physically meaningful (the constants are usually of the same order of magnitude). Theoretical aspects of such harmonic maps have been intensively studied, and a lot of results about existence, regularity, and singularities of minimizing or nonminimizing harmonic maps are now available. For example, mainly because of degree theory, maps from $\mathbb{R}^{3}$ into $S^{2}$ may have singularities that would correspond to defects in the structure of the nematic. From the numerical point of view, a few authors (Cohen et al. [4], Lin [11], Dean, Glowinski, and Li [5], Cohen, Lin, and Luskin [3], and Lin and Luskin [12]) have developed algorithms for finding stable solutions. However, mainly because of the lack of convexity of the constraint, the convergence of these algorithms has not always been established. The purpose of this paper is to give a new approach to this problem and to construct a very efficient algorithm in the harmonic mapping case. The proof of the convergence of this algorithm is also given.

2. The Oseen-Frank model. The liquid crystal phase is a transitional phase between liquid and solid. We may consider (at least for some specific liquid crystal) the material to be constituted of long molecules, one dimension of which is much larger than the others. Let $\Omega$ be a bounded domain of $\mathbb{R}^{3}$ in which some liquid crystal is embedded. Call for $x$ belonging to $\Omega, u(x)$ the mean direction of the molecules near $x$. This defines a map $u$ from $\Omega$ into the unit sphere $S^{2}$ of $\mathbb{R}^{3}$. Working with this

\footnotetext{
${ }^{*}$ Received by the editors March 7, 1994; accepted for publication (in revised form) December 15 , 1995.

http://www.siam.org/journals/sinum/34-5/26424.html

${ }^{\dagger}$ Centre de Mathématiques et de Leurs Applications, URA 1611, CNRS et Ecole Normale Supérieure de Cachan, 61 Avenue du Président Wilson, 94235 Cachan Cedex, France (alouges@cmla.ens-cachan.fr).
} 
background, Oseen and Frank associated the following energy with $u$ [13], [16], [7]:

$$
E(u)=\int_{\Omega} W(u, \nabla u) d x
$$

where the energy density is given by

$$
\begin{aligned}
W(u, \nabla u)= & \frac{1}{2}\left\{\left(K_{1}(\operatorname{div} u)^{2}+K_{2}(u \cdot \operatorname{curl}(u))^{2}+K_{3}|u \times \operatorname{curl}(u)|^{2}\right.\right. \\
& \left.+\left(K_{2}+K_{4}\right)\left(\operatorname{tr}\left[(\nabla u)^{2}\right]-(\operatorname{div} u)^{2}\right)\right\} .
\end{aligned}
$$

Here, the constants $K_{i}$ typically depend on the material and on the temperature. They are usually assumed to verify $K_{1}, K_{2}, K_{3}>0$ and $K_{2}>\left|K_{4}\right|$. Because physical stable configurations correspond to minimizers of $E$, we are interested in finding the configurations that agree to a nonconstant boundary data and minimize (even locally) the energy $E$. Mathematically, the problem reads

$$
\text { Minimize } E(u) \text { over } A_{n_{0}},
$$

where

$$
\begin{aligned}
A_{n_{0}} & \equiv H_{n_{0}}^{1}\left(\Omega, S^{2}\right) \\
& =\left\{u \in H^{1}\left(\Omega, R^{3}\right) ; u(x) \in S^{2} \text { a.e.; } u_{\mid \partial \Omega}=n_{0} \text { in the sense of trace }\right\} .
\end{aligned}
$$

The space $H^{1}$ is the usual Sobolev space, and $n_{0}$ is the boundary condition satisfying $n_{0}(x) \in S^{2}$ a.e. on $\partial \Omega$.

Two simplifications occur for problem (3):

- The fourth term in the energy is a null Lagrangian and depends only on the boundary data. This term is constant on $A_{n_{0}}$ and has no influence on the minimization problem.

- If we take $K_{1}=K_{2}=K_{3}=1$ and $K_{4}=0$, from $|u(x)|=1$ a.e., one easily computes

$$
E(u)=\frac{1}{2} \int_{\Omega}|\nabla u|^{2} d x
$$

which is the Dirichlet energy for maps between $\Omega$ and $S^{2}$. The problem becomes finding minimizing harmonic maps that take values into $S^{2}$.

In the following, we will restrict ourselves to this latter case (the general case will be the subject of a forthcoming work), and we give an explicit minimization algorithm well suited to this problem. The numerical implementation will be considered in the final sections.

3. An energy-decreasing algorithm. The question of finding numerically the solutions of this problem was first investigated by Lin [11] and Cohen et al. [4] (see also [3] and [12]). The following difficulties occur.

- Nonconvexity. The constraint $|u(x)|=1$ a.e. is not convex. Classical algorithms of minimization with convex constraints cannot be used directly.

- Nonregularity. The minimizers may not be continuous (actually, if the degree of the boundary data $n_{0}$ is different from 0 , no continuous map that takes values into $S^{2}$ belongs to $A_{n_{0}}$ ). 
- Nonuniqueness. Since $E$ doesn't change under transformations $u \longrightarrow Q^{T} \circ u \circ$ $Q$ where $Q$ is orthogonal, the solution may not be unique. Moreover, Rivière [14] recently showed the existence of infinitely many harmonic maps with the same boundary data $n_{0}$. At last, there exist harmonic maps (solutions to the Euler-Lagrange equations) that are not minimizing. Trying to directly solve the Euler-Lagrange equations of the problem is not suitable.

In [4] and [5] (and [3] for gradient methods), the authors gave an iterative procedure in which the iteration can be split into two steps (the relaxation method given in [12] or [3] does not fit into this context):

1. Let $u_{0}$ be an initial guess;

2. For $n=0 \ldots$ until convergence

2.1. Find $v_{n}$ such that $E\left(v_{n}\right) \leq E\left(u_{n}\right)$ where $v_{n}$ may not belong to $H^{1}\left(\Omega, S^{2}\right)$;

2.2. Set $u_{n+1}(x)=\frac{v_{n}(x)}{\left|v_{n}(x)\right|}$.

As far as step 2.1 is concerned, different methods can be used (e.g., gradient methods). The second step makes sure that the limit (if the algorithm converges) satisfies the constraint. The problem here is that the behavior of the energy during step 2.2 is unknown. In other words, the gain obtained during step 2.1 can be lost during the second step.

The algorithm we give is constructed in order to avoid this difficulty (actually, step 2.2 is also energy-decreasing) and accelerate the convergence. The complete construction follows three propositions.

Proposition 1. If $v \in H_{n_{0}}^{1}\left(\Omega, R^{3}\right)$ verifies $|v(x)| \geq 1$ a.e., then $v /|v|$ belongs to $H_{n_{0}}^{1}\left(\Omega, S^{2}\right)$, and moreover,

$$
\left|\nabla\left(\frac{v(x)}{|v(x)|}\right)\right|^{2} \leq|\nabla v(x)|^{2} \text { a.e., }
$$

from which we easily deduce that

$$
E\left(\frac{v}{|v|}\right) \leq E(v)
$$

Proof. This result can be shown by direct calculation. However, Serre [15] has remarked that it extends when $S^{2}$ is replaced by the boundary of any closed convex domain $C$. If $\Pi$ is the projection on $C$, one has

$$
|\nabla \Pi v(x)| \leq|\nabla v(x)| \text { a.e. } \quad \text { if } v(x) \notin C \text { a.e. }
$$

The proof of this property follows from the definition of the derivative and the contractivity of $\Pi$. Our case is only the particular case where $C=B^{3}$, the unit ball of $\mathbb{R}^{3}$.

This first proposition gives an explicit way of decreasing the energy during the renormalization stage (step 2.2). It remains to construct such a map $v$ which verifies the condition $|v(x)| \geq 1$ a.e. Given $u \in H_{n_{0}}^{1}\left(\Omega, S^{2}\right)$, the next two propositions allow the construction of $v$. 
Proposition 2. Let $K_{u}=\left\{w \in H_{0}^{1}\left(\Omega, R^{3}\right)\right.$ such that $w(x) . u(x)=0$ a.e. $\}$. If we write $v=u-w$, where $w$ belongs to $K_{u}$, then

$$
|v(x)|^{2}=|u(x)-w(x)|^{2}=1+|w(x)|^{2} \geq 1 \text { a.e. }
$$

Proposition 3. The problem

$$
\text { Minimize } \int_{\Omega}|\nabla(u-w)|^{2} d x \text { for } w \in K_{u}
$$

possesses a unique solution that we call $w(u)$.

Proof. $K_{u}$ is a convex (it is a linear space) of $H^{1}\left(\Omega, R^{3}\right)$, closed for the strong topology of $H^{1}$. The map $w$ is the projection of $u$ onto $K_{u}$ with respect to the usual distance on $H_{0}^{1}$.

Moreover, since $w \equiv 0$ belongs to $K_{u}$, one deduces

$$
\int_{\Omega}|\nabla(u-w(u))|^{2} d x \leq \int_{\Omega}|\nabla u|^{2} d x
$$

which ensures that the first step of the algorithm decreases the energy.

The algorithm then reads as follows:

1. Let $u_{0}$ be an initial guess;

2. For $n=0 \ldots$ until convergence

2.1. Solve the problem (9) and call $w_{n}=w\left(u_{n}\right)$ the solution;

2.2. Set $u_{n+1}=\frac{u_{n}-w_{n}}{\left|u_{n}-w_{n}\right|}$.

Two remarks can be made.

- The nonlinear nonconvex constraint $|u|=1$ a.e. has been transformed into the linear (easier-to-handle) one:

$$
u(x) \cdot w(x)=0 \text { a.e. }
$$

- From the construction itself, we easily deduce that

$$
\text { for all } n \geq 0, \quad E\left(u_{n+1}\right) \leq E\left(u_{n}-w_{n}\right) \leq E\left(u_{n}\right) .
$$

Since we do have control of the energy during the algorithm, we obtain the following result concerning the convergence of this algorithm.

THEOREM 1. The algorithm $(A)$ converges in the sense that $\left(u_{n}\right)$ (up to a subsequence) weakly converges in $H^{1}\left(\Omega, R^{3}\right)$ to a harmonic map $u_{\infty} \in H_{n_{0}}^{1}\left(\Omega, S^{2}\right)$ where $n_{0}$ is the boundary data of $u_{0}$. Moreover, the entire sequence $\left(w_{n}\right)_{n \geq 0}$ strongly converges to 0 in $H_{0}^{1}\left(\Omega, R^{3}\right)$.

Proof. We first use the following lemma in order to estimate the energy decrease during the step 2.1.

LEMMA 1. We have for all $n \geq 0$

$$
\int_{\Omega}\left|\nabla u_{n}\right|^{2} d x=\int_{\Omega}\left|\nabla\left(u_{n}-w_{n}\right)\right|^{2} d x+\int_{\Omega}\left|\nabla w_{n}\right|^{2} d x .
$$

Proof. It suffices to write the variational formulation of the problem (9):

$$
\int_{\Omega} \nabla\left(u_{n}-w_{n}\right) \cdot \nabla \Psi d x=0 \forall \Psi \in K_{u_{n}}
$$

Taking $\Psi=w_{n}$ gives the desired result. 
Now, since the step (2.2) is also energy decreasing, we may write

$$
\int_{\Omega}\left|\nabla u_{n+1}\right|^{2} d x \leq \int_{\Omega}\left|\nabla\left(u_{n}-w_{n}\right)\right|^{2} d x=\int_{\Omega}\left|\nabla u_{n}\right|^{2} d x-\int_{\Omega}\left|\nabla w_{n}\right|^{2} d x,
$$

from which we deduce

$$
\int_{\Omega}\left|\nabla w_{n}\right|^{2} d x \leq \int_{\Omega}\left|\nabla u_{n}\right|^{2} d x-\int_{\Omega}\left|\nabla u_{n+1}\right|^{2} d x .
$$

Summing (12) from $n=0$ to $n=N$ leads to

$$
\sum_{n=0}^{N} \int_{\Omega}\left|\nabla w_{n}\right|^{2} d x \leq \int_{\Omega}\left|\nabla u_{0}\right|^{2} d x,
$$

and the series

$$
\sum_{n \geq 0} \int_{\Omega}\left|\nabla w_{n}\right|^{2} d x
$$

is convergent. The conclusion that

$$
w_{n} \longrightarrow 0 \text { strongly in } H^{1}
$$

follows immediately.

It remains to prove that (up to a subsequence) $\left(u_{n}\right)$ weakly converges to a harmonic map $u_{\infty}$ in $H^{1}$. But we have, for all $n \geq 0$,

$$
\int_{\Omega}\left|\nabla u_{n}\right|^{2} d x \leq \int_{\Omega}\left|\nabla u_{0}\right|^{2} d x
$$

That means that the sequence $\left(u_{n}\right)$ is bounded in $H^{1}$. We may extract a subsequence (that we still denote by $\left(u_{n}\right)$ ) which converges weakly in $H^{1}$, strongly in $L^{2}$, and a.e. to a map called $u_{\infty}$. Since $u_{n}$ belongs to $H_{n_{0}}^{1}\left(\Omega, S^{2}\right)$ and since this set is closed under weak- $H^{1}$ convergence, we deduce that $u_{\infty}$ belongs to $H_{n_{0}}^{1}\left(\Omega, S^{2}\right)$. The last assertion is that $u_{\infty}$ is a harmonic map. In order to solve this question, we need to write the Euler-Lagrange equations of which $w_{n}$ is a solution:

$$
\int_{\Omega} \nabla\left(u_{n}-w_{n}\right) \cdot \nabla \Psi d x=0 \forall \Psi \in K_{u_{n}} .
$$

Taking $\Psi$ under the form $\Psi=\phi \times u_{n}$, where $\phi \in C_{0}^{\infty}\left(\Omega, R^{3}\right)$, gives

$$
\int_{\Omega} \nabla\left(u_{n}-w_{n}\right) \cdot\left(\nabla \phi \times u_{n}+\phi \times \nabla u_{n}\right) d x=0 .
$$

Expanding this expression gives

$$
\int_{\Omega} \nabla u_{n} \cdot \nabla \phi \times u_{n}-\nabla w_{n} \cdot\left(\nabla \phi \times u_{n}+\phi \times \nabla u_{n}\right) d x=0
$$

or

$$
\int_{\Omega} \nabla \phi \cdot u_{n} \times \nabla u_{n}-\nabla \phi \cdot u_{n} \times \nabla w_{n}-\phi \cdot \nabla u_{n} \times \nabla w_{n} d x=0 .
$$

This means that

$$
\operatorname{div}\left(u_{n} \times \nabla\left(u_{n}-w_{n}\right)\right)=\nabla w_{n} \times \nabla u_{n}
$$


in the sense of distributions. Using the facts that

$$
\begin{gathered}
u_{n} \rightarrow u_{\infty} \text { weakly in } H^{1}, \\
u_{n} \rightarrow u_{\infty} \text { strongly in } L^{2}, \\
w_{n} \rightarrow 0 \text { strongly in } H^{1},
\end{gathered}
$$

we can pass to the limit and write

$$
\operatorname{div}\left(u_{\infty} \times \nabla u_{\infty}\right)=0
$$

in the sense of distributions, which is equivalent to the fact that $u_{\infty}$ is a harmonic map (as remarked by Chen [2]).

4. A few remarks about the map $u \rightarrow w(u)$. In this section, we would like to make a few remarks concerning the map

$$
\begin{aligned}
w: H^{1}\left(\Omega, S^{2}\right) & \longrightarrow H_{0}^{1}\left(\Omega, R^{3}\right), \\
u & \longrightarrow w(u),
\end{aligned}
$$

where $w(u)$ is the solution of the problem (9). Lemma 1 gives

$$
\int_{\Omega}|\nabla u|^{2} d x=\int_{\Omega}|\nabla(u-w(u))|^{2} d x+\int_{\Omega}|\nabla w(u)|^{2} d x
$$

and moreover, $w(u)$ is a solution of the associated variational formulation

$$
\int_{\Omega} \nabla(u-w(u)) \cdot \nabla \Psi d x=0 \forall \Psi \in K_{u} .
$$

We now have the following remark.

Remark 1. The map $w(u)$ is the unique solution of the variational formulation (13) in $K_{u}$.

Proof. We already know that $w(u)$ is a solution. In order to prove the uniqueness, consider two solutions $w_{1}$ and $w_{2}$ in $K_{u}$ of (13). We may write (since $w_{1}-w_{2} \in K_{u}$ )

$$
\int_{\Omega} \nabla\left(u-w_{1}\right) \cdot \nabla\left(w_{1}-w_{2}\right) d x=0
$$

and

$$
\int_{\Omega} \nabla\left(u-w_{2}\right) \cdot \nabla\left(w_{1}-w_{2}\right) d x=0 .
$$

Subtracting these two equalities gives

$$
\int_{\Omega}\left|\nabla\left(w_{1}-w_{2}\right)\right|^{2} d x=0
$$

which means

$$
w_{1}=w_{2}
$$

since $w_{1}$ and $w_{2}$ belong to $H_{0}^{1}$.

Remark 2. The map $u$ is a harmonic mapping if and only if $w(u)=0$. 
Proof. If 0 is the solution of (13), we have

$$
\int_{\Omega} \nabla u \cdot \nabla \Psi d x=0 \quad \forall \Psi \in K_{u} .
$$

By taking trial functions $\Psi$ under the form $\Psi=\phi \times u$, where $\phi \in C_{0}^{\infty}\left(\Omega, R^{3}\right)$, we find that

$$
\operatorname{div}(u \times \nabla u)=0
$$

in the sense of distributions, which is equivalent to the fact that $u$ is a harmonic mapping (see [2]). Conversely, from (14), we may deduce (13) with $w(u)=0$ by a density argument.

5. Implementations of the algorithm. Here we give three different ways to numerically implement the algorithm. The first is a saddle-point technique, whereas the second is closely related to a relaxation method for solving the Poisson problem. The third appears to be a conjugate gradient method. Some comparisons in terms of computation times between these implementations are given.

5.1. Discretization. We use finite differences. Following [11] and [4], we call $C=[0,1]^{3}$ the unit cube of $\mathbb{R}^{3}$. If $h=\frac{1}{M}$ is the space-step, we approximate a map $u: C \longrightarrow S^{2}$ by the values at the edges of the mesh

$$
u_{i j k}^{h} \approx u(i h, j h, k h) \text { for } 0 \leq i, j, k \leq M .
$$

The (approximated) energy is simply given by

$$
\begin{aligned}
E^{h}\left(u_{i j k}^{h}\right)= & \frac{1}{2} \sum_{i, j, k=0}^{M-1}\left[\left|\frac{u_{i+1, j, k}^{h}-u_{i j k}^{h}}{h}\right|^{2}+\left|\frac{u_{i, j+1, k}^{h}-u_{i j k}^{h}}{h}\right|^{2}\right. \\
& \left.+\left|\frac{u_{i, j, k+1}^{h}-u_{i j k}^{h}}{h}\right|^{2}\right] h^{3},
\end{aligned}
$$

and we look for a minimizer of the energy $E^{h}$ in the discretized space:

$$
\begin{aligned}
& A_{n_{0}}^{h}=\left\{u_{i j k}^{h} ;\left|u_{i j k}^{h}\right|=1 \text { for all } 0 \leq i, j, k \leq M\right. \\
&\text { and } \left.u_{i j k}^{h}=n_{0, i j k} \text { for all } i, j, k \operatorname{such} \text { that }(i h, j h, k h) \in \partial C\right\} .
\end{aligned}
$$

It is also necessary to define the space of variations (the discretized version of $K_{u}$ )

$$
\begin{aligned}
K_{u_{i j k}^{h}}^{h}= & \left\{w_{i j k}^{h} ; w_{i j k}^{h} \cdot u_{i j k}^{h}=0 \text { for all } 0 \leq i, j, k \leq M\right. \\
& \text { and } \left.w_{i j k}^{h}=0 \text { for all } i, j, k \text { such that }(i h, j h, k h) \in \partial C\right\} .
\end{aligned}
$$

With all these notations, the discretized algorithm follows directly:

1. Let $u_{0, i j k}^{h}$ be an initial guess;

2. For $n=0 \ldots$ until convergence

2.1. Minimize $E^{h}\left(u_{n, i j k}^{h}-w_{i j k}^{h}\right)$ over $K_{u_{n, i j k}^{h}}^{h}$ and call $w_{n, i j k}^{h}$ the solution;

2.2. Set $u_{n+1, i j k}^{h}=\frac{u_{n, i j k}^{h}-w_{n, i j k}^{h}}{\left|u_{n, i j k}^{h}-w_{n, i j k}^{h}\right|}$. 
Step 2.2 is still energy-decreasing because of the Lipschitz property of the projection $\Pi$ onto the unit sphere (see Proposition 1). Hence, the only remaining problem is to solve step 2.1, that is,

$$
\text { Minimize } E^{h}\left(u_{n, i j k}^{h}-w_{i j k}^{h}\right) \text { over } w_{i j k}^{h} \in K_{u_{n, i j k}^{h}}^{h} .
$$

We give three different methods for the resolution of (15) and the corresponding results.

5.2. A saddle-point technique. In order to solve the problem (15), it seems natural to introduce the following augmented Lagrangian:

$$
\begin{aligned}
L_{h}\left(w_{i j k}^{h}, \omega_{i j k}^{h}\right)=E^{h}\left(u_{i j k}^{h}-w_{i j k}^{h}\right) & +\frac{r}{2} \sum_{i, j, k=0}^{M}\left(u_{i j k}^{h} \cdot w_{i j k}^{h}\right)^{2} \\
& +\sum_{i, j, k=0}^{M} \omega_{i j k}^{h}\left(u_{i j k}^{h} \cdot w_{i j k}^{h}\right) .
\end{aligned}
$$

Since $L_{h}(w, \omega)$ can be rewritten as

$$
L_{h}(w, \omega)=\frac{1}{2}(A w, w)-(b, w)+(\omega, B w)+\frac{r}{2}|B w|^{2}+C,
$$

where $A$ is a positive definite matrix, $b$ is a vector, $B$ is the constraint, and $C$ is a constant, it is easily seen (see [6, p. 2]) that this Lagrangian has a unique saddle-point (the unicity comes from the fact that the map $w \rightarrow B w$ is onto)

$$
\left(\bar{w}_{i j k}^{h}, \bar{\omega}_{i j k}^{h}\right) \in R^{3(M+1)^{3}} \times R^{(M+1)^{3}},
$$

which satisfies

$$
L_{h}\left(\bar{w}_{i j k}^{h}, \bar{\omega}_{i j k}^{h}\right)=\inf _{w \in R^{3(M+1)^{3}}} \sup _{\omega \in R^{(M+1)^{3}}} L_{h}(w, \omega) .
$$

That means

$$
\text { - } u_{i j k}^{h} \cdot \bar{w}_{i j k}^{h}=0 \text { for all } 0 \leq i, j, k \leq M \text {, }
$$

- $\bar{w}_{i j k}^{h}$ minimizes $E^{h}\left(u_{i j k}^{h}-w_{i j k}^{h}\right)$ with respect to this constraint.

We have implemented a standard Uzawa method for finding the saddle-point (see $[6$, p. 21]). With the notations above, and setting

$$
A_{r}=A+r B^{t} B
$$

this method is as follows:

$$
\left[\begin{array}{l}
\text { Start with } g_{0}, w_{0}=A_{r}^{-1}\left(b-B^{t} g_{0}\right) \\
\text { For } n=0 \ldots \text { until convergence }
\end{array}\right.
$$




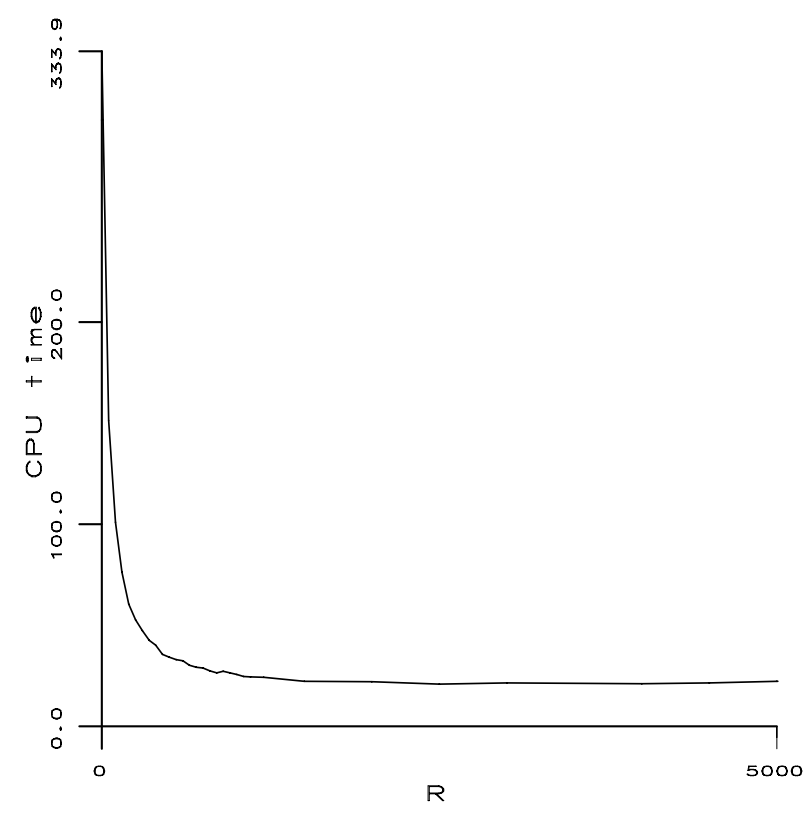

FIG. 1. CPU time for augmented Lagrangian technique with respect to the augmentation parameter $r$ on a $21^{3}$ grid.

As usual, the rate of convergence of the algorithm is closely related to the value of $r$ (the augmentation parameter). The bigger $r$ is, the faster (in terms of iterations) the saddle-point is reached, but the more ill conditioned $A_{r}$ is. As the inversion of the matrix $A_{r}$ at each iteration is done by a conjugate gradient method, very big values of $r$ slow down the total time for finding the saddle-point. In Fig. 1 we see the time used for the resolution of the saddle-point for different values of $r$. Here the mesh is $21 \times 21 \times 21$ and good values of $r$ are between 2000 and 5000 .

5.3. A relaxation method. This method is inspired by the resolution of the Poisson problem with Dirichlet boundary conditions:

$$
\left\{\begin{array}{l}
\Delta u=f \text { on } \Omega \\
u=0 \text { on } \partial \Omega
\end{array}\right.
$$

More precisely, when we want to solve the Euler-Lagrange equations of problem (15), we are led to solve

$$
\left\{\begin{array}{c}
\Delta^{h}\left(u_{i j k}^{h}-w_{i j k}^{h}\right)=\omega_{i j k} u_{i j k}^{h} \\
w_{i j k}^{h}=0 \text { if }(i h, j h, k h) \in \partial \Omega
\end{array}\right.
$$

where $\Delta^{h}$ is the classical five-point discretization of the Laplace operator in finite differences. Calling

$$
b\left(v_{i j k}^{h}\right)=\left[v_{i+1, j, k}^{h}+v_{i-1, j, k}^{h}+v_{i, j+1, k}^{h}+v_{i, j-1, k}^{h}+v_{i, j, k+1}^{h}+v_{i, j, k-1}^{h}\right],
$$


we can rewrite this in the form

$$
\left(b\left(u_{i j k}^{h}\right)-b\left(w_{i j k}^{h}\right)-6\left(u_{i j k}^{h}-w_{i j k}^{h}\right)\right) \times u_{i j k}^{h}=0 .
$$

We will give a procedure to iteratively solve these equations. Keeping in mind that

$$
w_{i j k}^{h} \perp u_{i j k}^{h},
$$

we deduce

$$
w_{i j k}^{h}=\mu_{i j k}\left(b\left(u_{i j k}^{h}\right)-b\left(w_{i j k}^{h}\right)-\left(\left(b\left(u_{i j k}^{h}\right)-b\left(w_{i j k}^{h}\right)\right) \cdot u_{i j k}^{h}\right) u_{i j k}^{h}\right) .
$$

Putting this expression into

$$
\left(b\left(u_{i j k}^{h}\right)-b\left(w_{i j k}^{h}\right)-6\left(u_{i j k}^{h}-w_{i j k}^{h}\right)\right) \cdot w_{i j k}^{h}=0
$$

leads to

$$
\mu_{i j k}=-\frac{1}{6}
$$

Thus, (17) may be rewritten as

$$
\begin{aligned}
w_{i j k}^{h} & =\frac{1}{6}\left[\left(\left(b\left(u_{i j k}^{h}\right)-b\left(w_{i j k}^{h}\right)\right) \cdot u_{i j k}^{h}\right) u_{i j k}^{h}-\left(b\left(u_{i j k}^{h}\right)-b\left(w_{i j k}^{h}\right)\right)\right] \\
& =\frac{1}{6} u_{i j k}^{h} \times\left(u_{i j k}^{h} \times\left(b\left(u_{i j k}^{h}\right)-b\left(w_{i j k}^{h}\right)\right)\right) .
\end{aligned}
$$

Let us choose a relaxation parameter $\mu \in(0,2)$. We start the iterative procedure with $w_{0, i j k}^{h}=0$. If we introduce the lexicographical order $\ll$ on $\mathbb{R}^{3}$ defined by

$$
\begin{gathered}
(i, j, k) \ll(l, m, n) \text { if and only if } i<l \text { or } i=l \text { and } j<m \\
\text { or } i=l \text { and } j=m \text { and } k<n,
\end{gathered}
$$

we can write the following algorithm:

For $L=1 \ldots$ until convergence

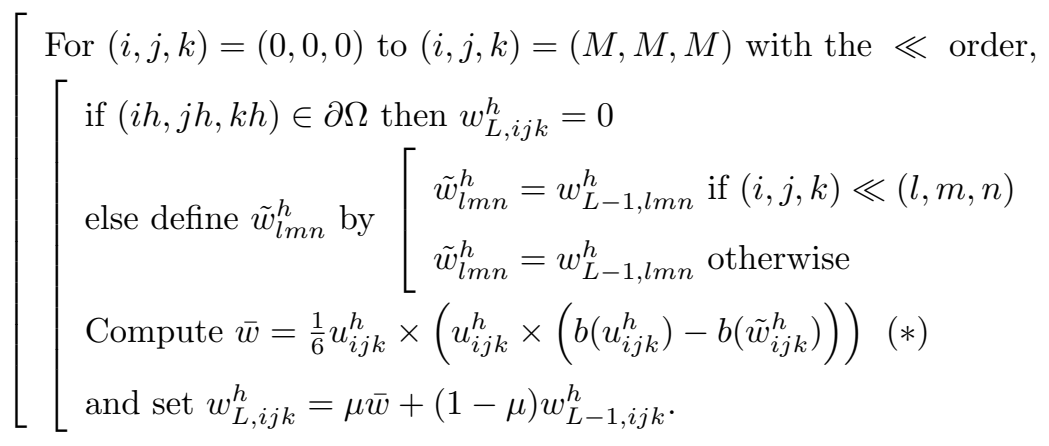

We remark that this algorithm is exactly the same as the one used for solving the Poisson problem (16) with a relaxation technique applied to a Gauss-Seidel iteration. The basic calculation $(*)$ here stands for

$$
\bar{w}=\frac{1}{6} b\left(\tilde{w}_{i j k}^{h}\right),
$$




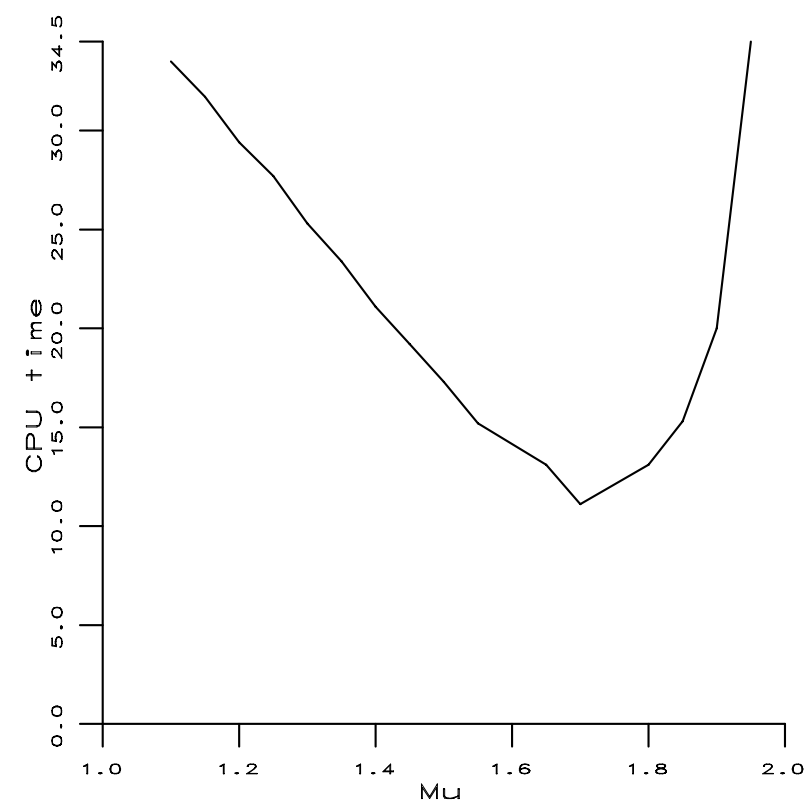

FIG. 2. CPU time for the relaxation method with respect to $\mu$, on a $21^{3}$ grid. The optimal value of $\mu$ is around 1.7 .

which we would have found during the resolution of (16). Notice that the relaxation technique developed by Lin and Luskin [12] is quite different, since they try to solve the nonlinear constrained problem directly. Here, the constraint has been linearized, and this relaxation method is an easy adaptation of the one used to solve (16).

The advantage of this method is of course its ease of implementation. Moreover, the constraint is accurately verified (we search directly for $w^{h}$ in $K_{u^{h}}$ ), instead of being verified at the limit. The problem comes from finding the best value of $\mu$. Figure 2 shows the CPU time used for solving the same problem as before (see Fig. 1 for the Uzawa method) with various values of $\mu$ between 1 and 2 (values less than 1 give higher computing time).

6. A conjugate gradient technique. As we might expect from the last section, we can write a conjugate gradient algorithm for solving the problem (15). As before, this method is very different from the one used by Cohen, Lin, and Luskin [3] in the sense that we solve a linear problem as described in the following remark.

Remark 3. Suppose we want to minimize

$$
F(X)=\frac{1}{2}(A X, X)-(b, X),
$$

where (.,.) is the inner product on $\mathbb{R}^{N}, A$ is a positive definite $N \times N$ matrix, $b$ is a vector in $\mathbb{R}^{N}$, and $X \in \mathbb{R}^{N}$, subject to the constraint

$$
B X=0 .
$$

Then $X$ may be obtained by applying a conjugate gradient procedure to the functional

$$
\tilde{F}(X)=\frac{1}{2}(\pi A \pi X, X)-(\pi b, X)
$$


TABLE 1

\begin{tabular}{|c|c|c|}
\hline \multicolumn{3}{|c|}{$\begin{array}{c}\text { CPU time for solving problem } \\
\text { on a Sparc2 computer }\end{array}$} \\
\hline & $15^{3}$ grid & $21^{3}$ grid \\
\hline Lagrangian method & $\approx 8.8 s$ & $\approx 21.1 s$ \\
\hline Relaxation method & $\approx 4.7 s$ & $\approx 11.1 s$ \\
\hline Conjugate gradient & $\approx 3.7 s$ & $\approx 11.3 s$ \\
\hline
\end{tabular}

where $\pi$ stands for the orthogonal projector onto the linear space

$$
K=\left\{X \in \mathbb{R}^{N} \text { such that } B X=0\right\},
$$

provided the algorithm is started with $X_{0} \in K$.

The proof of this remark is obvious and left to the reader. It is also clear (since $\tilde{F}(X)=\tilde{F}(\pi X)$ ) that any minimization procedure that converges to a minimum $X_{\infty}$ of $\tilde{F}$ will give the correct solution to (18), $\bar{X}=\pi X_{\infty}$.

Setting $\pi^{h}$ to be the projector onto the plane orthogonal to $u_{i j k}$,

$$
\pi^{h} w_{i j k}=w_{i j k}-\left(w_{i j k} . u_{i j k}\right) u_{i j k},
$$

we implemented the classical conjugate gradient applied to the functional (18):

Start with $w_{0} \in K_{u_{n, i j k}}, r_{0}=-\pi^{h} \Delta^{h}\left(u_{i j k}^{h}-w_{0, i j k}\right), p_{0}=r_{0} ;$

For $n=0 \ldots$ until convergence

$$
\left[\begin{array}{l}
\alpha_{n}=\frac{\left(r_{n}, p_{n}\right)}{\left(p_{n},-\pi^{h} \Delta^{h} p_{n}\right)} \\
w_{n+1, i j k}=w_{n, i j k}+\alpha_{n} p_{n} \\
r_{n+1}=r_{n}+\alpha_{n} \pi^{h} \Delta^{h} p_{n} \\
\beta_{n}=\frac{\left|r_{n+1}\right|^{2}}{\left|r_{n}\right|^{2}} \\
p_{n+1}=r_{n+1}+\beta_{n} p_{n}
\end{array}\right.
$$

In other words, the possibility of writing a conjugate gradient method comes from the explicit knowledge of $\pi^{h}$. Indeed, in our problem, $\pi^{h}$ is the pointwise projection onto the plane orthogonal to $u$. Thus, this method appears to be "equivalent" (in terms of complexity) to one iteration of the Uzawa method (for the augmented Lagrangian).

7. Comparison of the algorithms. We described three algorithms in the preceding section. We give here (see also Table 1) a short comparison in terms of CPU times on two cases. The first case is on a $15^{3}$ grid and the second is on a $21^{3}$ grid. For both the Uzawa method and the relaxation method, we give the best performance obtained. The same problem has been chosen for comparing the three algorithms, on the same grid. The initial value for $w_{i j k}$ is 0 , and the algorithms stop when the Euclidean norm of the residual has been reduced by a factor of $10^{-4}$. The map taken for $u^{h}$ is of degree 1 with displaced singularity, which is drawn in Fig. 3.

As expected, the augmented Lagrangian technique (even with the optimal parameter $r$ ) is much more costly than either relaxation or conjugate gradient techniques. 


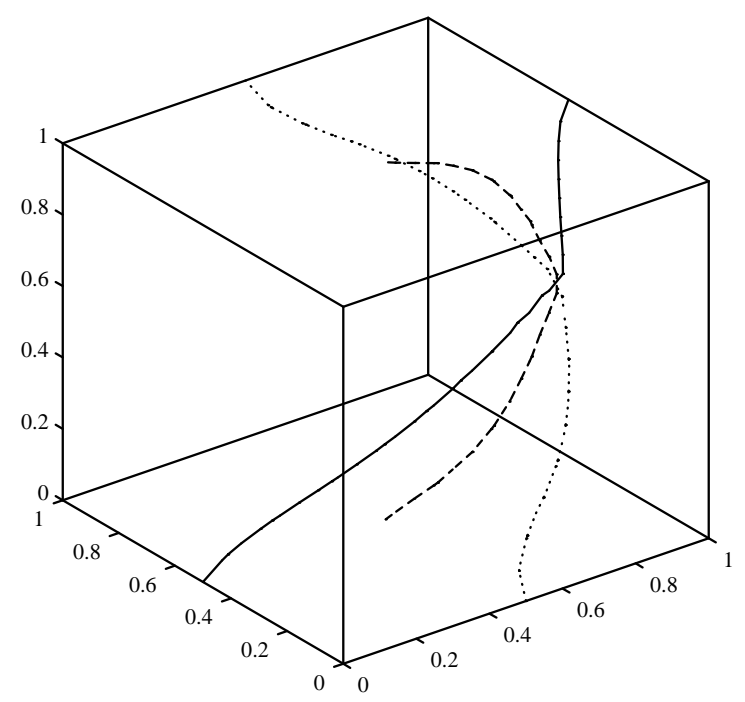

FIG. 3. Initial map presenting a degree 1 singularity and which agrees with $x /|x|$ on the boundary.

Moreover, choosing bad values for the Lagrangian and relaxation methods can be dramatic. As the fastest method is the conjugate gradient where no parameter needs to be adjusted, we made the most of the computations below with this method.

8. Using the fibers for representing the solution. In order to "draw" the solution, we decided to draw some of its fibers. We will briefly discuss the advantage of using the fibers. (In [8] and [9] Gulliver studied the fibers from the mathematical point of view.)

DEFINITION 1. Let $u \in H_{n_{0}}^{1}\left(\Omega, S^{2}\right)$; we call

$$
F_{u}(s)=u^{-1}(\{s,-s\})
$$

the fiber of $u$ associated with a vector $s \in S^{2}$.

Generically, since $\Omega$ is of dimension 3, these fibers are curves in $\Omega$.

Example. If we consider the map

$$
\begin{gathered}
u_{*}: \Omega \longrightarrow S^{2}, \\
x \longrightarrow \frac{x}{|x|},
\end{gathered}
$$

the fibers of $u_{*}$ are obviously straight lines that are crossing each other at 0 . In the preceding example, the fibers cross each other exactly where the map has a singularity. Conversely, if two fibers cross each other at a point $x$ in $\Omega$, then there is a singularity at $x$ (since the map has to take different values at $x$ ). More precisely, if $x$ is a singularity of degree $k$, for $u$, since $u$ maps " $k$ times" the sphere $S^{2}$ locally around $x$ (that is, on any sufficiently small sphere around $x$ ) from the definition of the degree, then we expect any fiber of $u$ to go at least $k$ times through $x$. From the above remarks, and taking into account the result by Brezis, Coron, and Lieb [1] that locally minimizing harmonic maps may have only singularities of degree \pm 1 , drawing few fibers (three fibers are usually sufficient) immediately allows the localization of the singularities. 


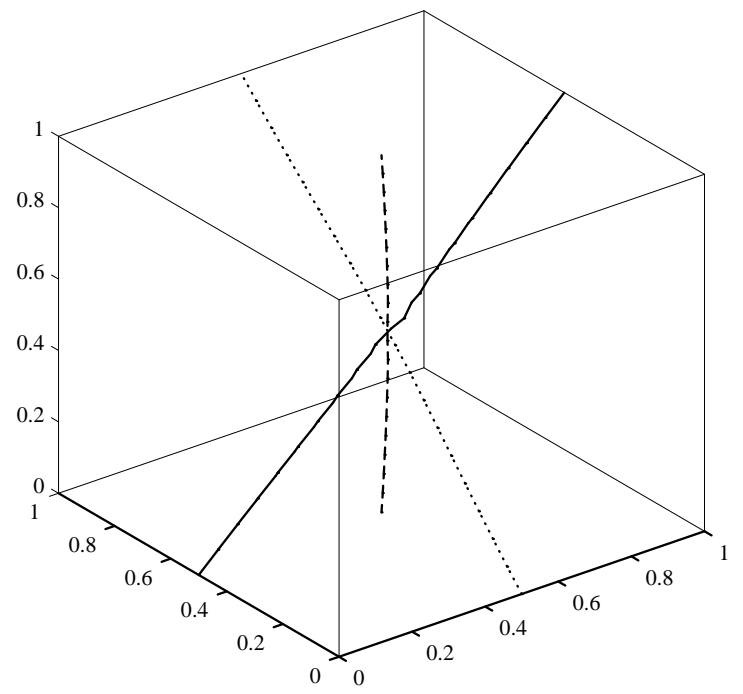

FIG. 4. The algorithm converges to a final map which possesses straight fibers. This is $x \longrightarrow$ $x /|x|$.

\section{Numerical results.}

9.1. The map $x /|x|$ is minimizing. The first example treated is to verify the minimizing property of the map

$$
\begin{aligned}
u_{*}: \Omega & \longrightarrow S^{2}, \\
x & \longrightarrow \frac{x}{|x|} .
\end{aligned}
$$

There exist many proofs of this result (one of the simplest is due to Lin [10]).

Figure 3 shows the initial data, which is a map with one singularity of degree 1 displaced on the right of the cube and which agrees with $u_{*}$ on $\partial \Omega$. After a few iterations of the algorithm (see Fig. 4), the singularity moved to the center and the fibers are now straight lines. This means that this final map is $u_{*}$. Remark that the boundary condition did not change, since the fibers still cross the boundary at the same places. Figure 5 shows the energy diagram during these iterations.

9.2. The singularities of degree greater than 1 are unstable. This has been proved by Brezis, Coron, and Lieb [1] after having seen the numerical results of Cohen et al. [4]. The result is that minimizing harmonic maps may only have singularities of degree \pm 1 . Conversely, singularities of degree $2,3, \ldots$ are unstable. The best way to construct a singularity of degree $n$ is to consider the map

$$
\begin{aligned}
u_{\omega}: \Omega \longrightarrow S^{2}, \\
\quad x \longrightarrow \pi_{s}^{-1} \circ \omega \circ \pi_{s}\left(\frac{x}{|x|}\right),
\end{aligned}
$$

where $\pi_{s}$ is the stereographic projection from $S^{2}$ into the complex plane $C$ and $\omega$ is a map from $C$ to $C$. If $\omega$ is holomorphic, then the map $u_{\omega}$ is harmonic. Moreover, $u_{\omega}$ has only one singularity in 0 , and if, for example, $\omega$ is polynomial of degree $n$, then this singularity is of degree $n$. 


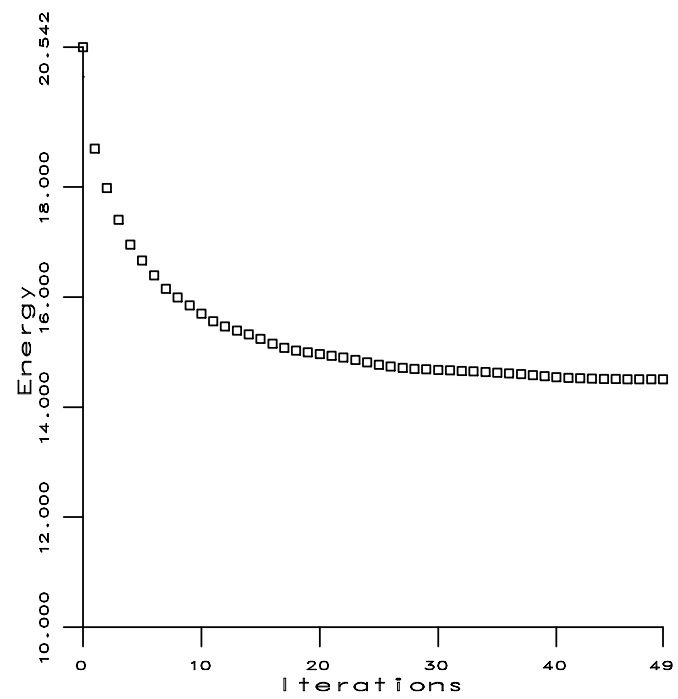

FIG. 5. Energy versus iterations diagram for the degree 1 singularity.

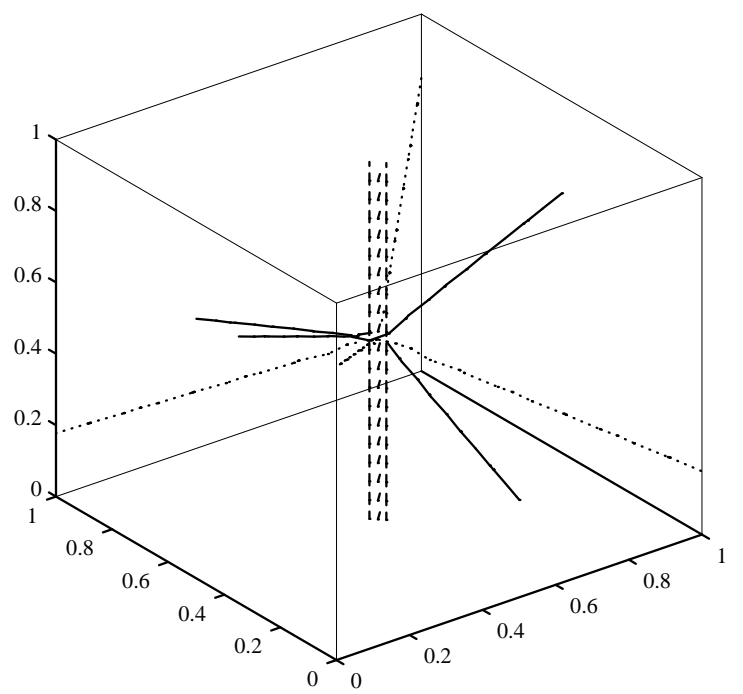

FIG. 6. Initial map with a degree 2 singularity.

We have decided to start the algorithm with a map of the form $u_{\omega}$ with

- $\omega(z)=z^{2}$,

- $\omega(z)=z^{3}$.

In each case we expect the singularity to "explode" into a few singularities, each of degree 1.

As far as the first case is concerned $\left(\omega(z)=z^{2}\right)$, we have represented some fibers of the discretized map $u_{\omega}^{h}$ in Fig. 6. Due to the discretization, the singularity is delocalized in the center cell. After a few iterations (Fig. 7), the algorithm finds a "stable" map which possesses 2 singularities of degree +1 . Numerically, degree 2 singularities are also unstable. The energy diagram is presented in Fig. 8, where it can 


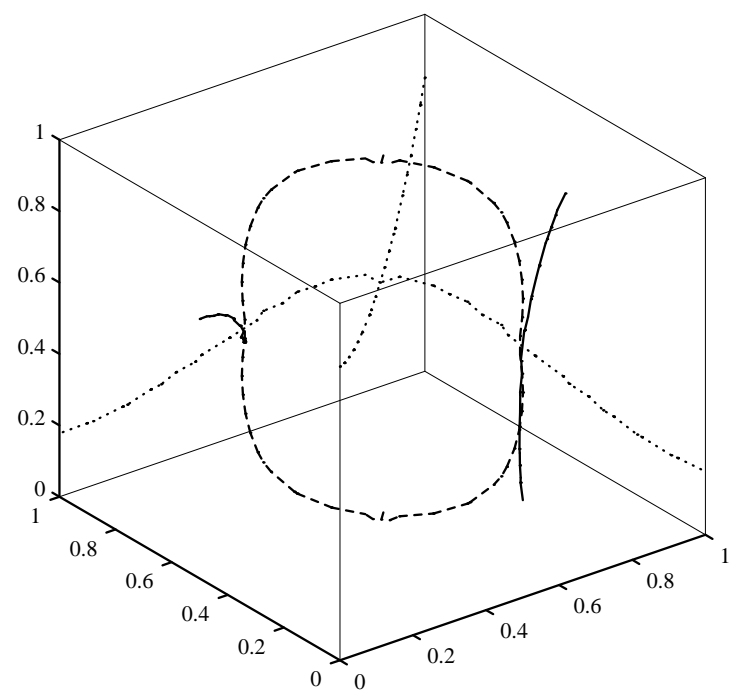

FiG. 7. Final map with two degree 1 singularities.

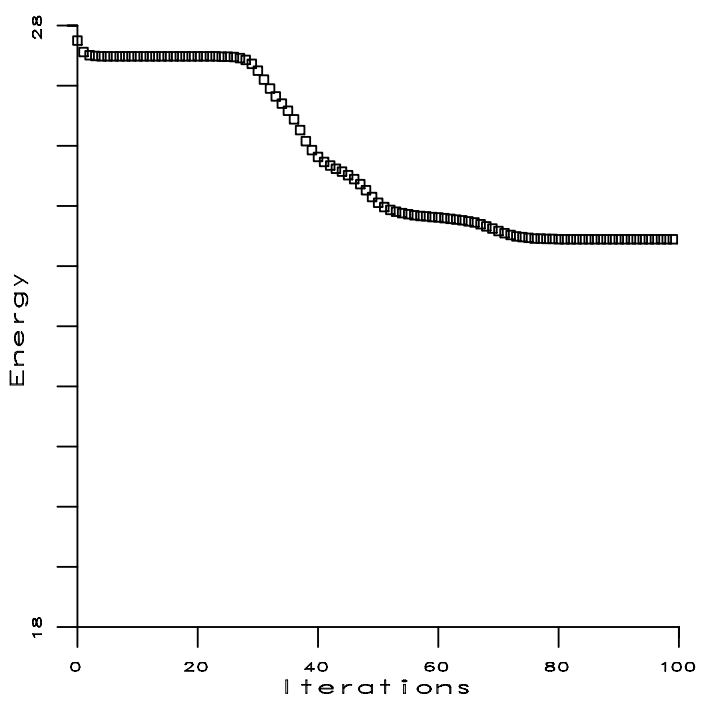

FIG. 8. Energy versus iterations for the creation of two degree 1 singularities.

be seen that the algorithm has some difficulties lowering the energy at the beginning. The reason is that since the initial guess is a harmonic map, the first computed descent directions are almost 0 . However, the algorithm is stable enough to slowly decrease the energy and escape from this critical point. Another possibility for testing the stability of the initial mapping would have been to perturb it.

The second case is a very special one; actually, the first algorithm (augmented Lagrangian technique) led to a map with three singularities of degree 1 (see Figs. 9 and 10 for initial and limit maps). The second and third (much faster) algorithms found a map with five singularities, four of degree 1 , and one of degree -1 , in the 


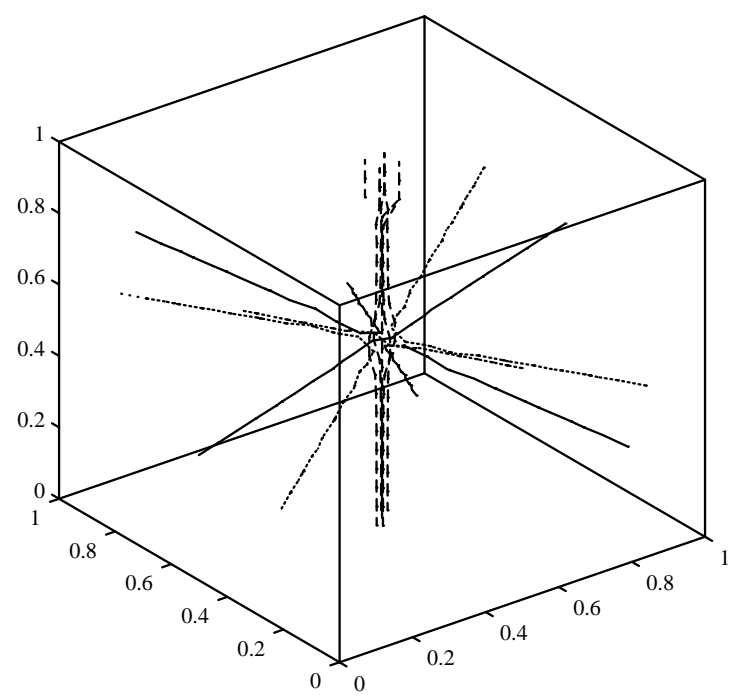

FIG. 9. Initial map with a degree 3 singularity.

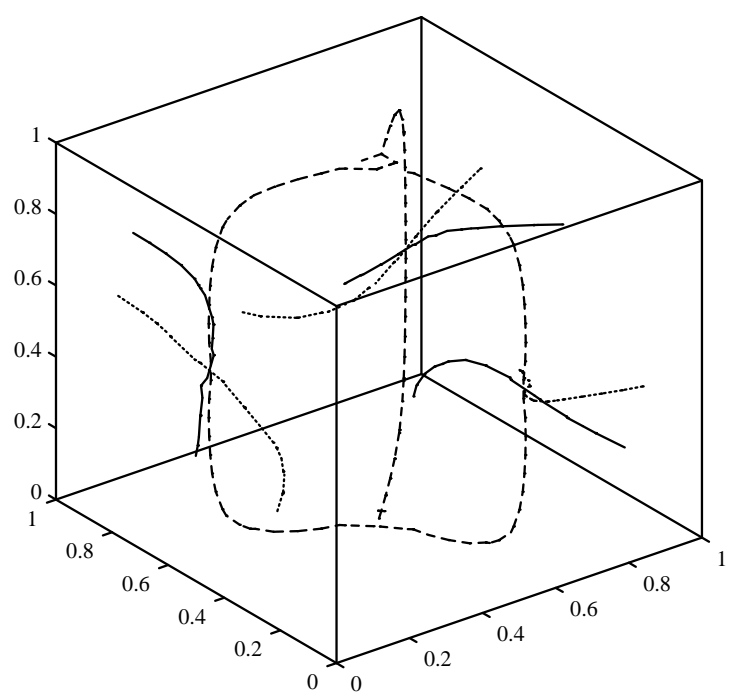

FIG. 10. Final map with three degree 1 singularities obtained with the Uzawa method.

center, conserving the total degree of the map. The main reason for this strange result is that the constraint is very well satisfied; it is also due to the symmetry of the problem (see Figs. 11, 12, and 13 for a few iterates). This is the only case where a different result has been found when running the different algorithms. The second minimizer is of energy greater than the first one, indicating that we found a local minimizer of the energy. (Even the augmented Lagrangian algorithm started with this map as an initial guess did not succeed in decreasing the energy.) 


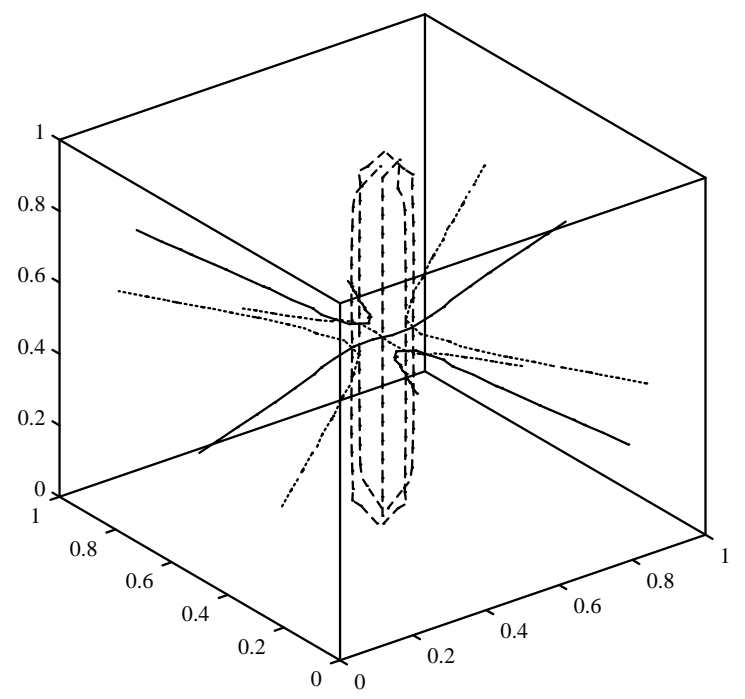

FIG. 11. After 10 iterations of the relaxation or conjugate gradient methods.

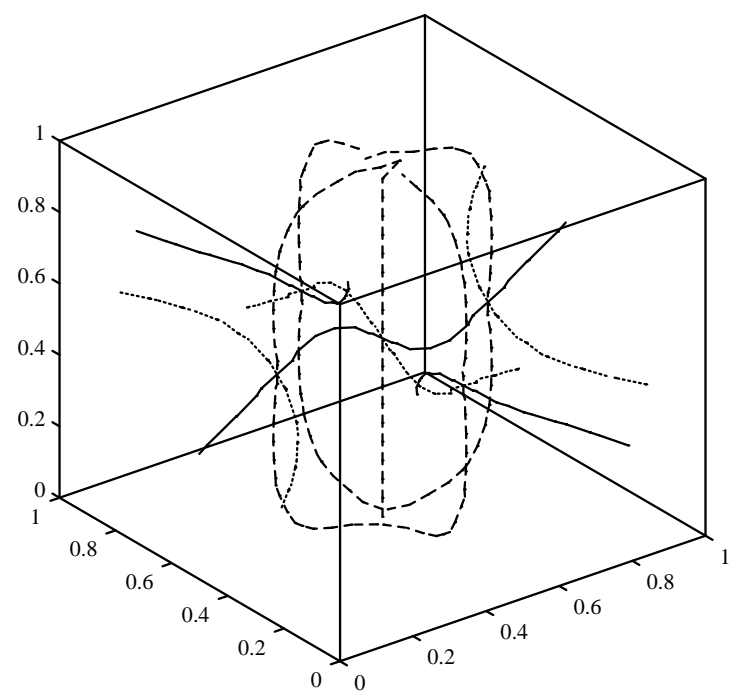

FIG. 12. After 50 iterations of the relaxation or conjugate gradient methods.

10. Conclusion. We have constructed a very efficient algorithm well suited for finding minimizing $S^{2}$-valued harmonic maps. From some properties of the construction, we have been able to prove the convergence of this method. After discretization, we obtained a numerical version of this algorithm which possesses a very good rate of convergence (but each iteration requires the resolution of a linear system). Unfortunately, generalizing this process to the more complicated case of nonequal constants $K_{i}$ does not seem that easy. Since the energy is no longer quadratic, the problem (15) is nonlinear and may not have a unique solution. Moreover, the renormalization step may increase the energy. 


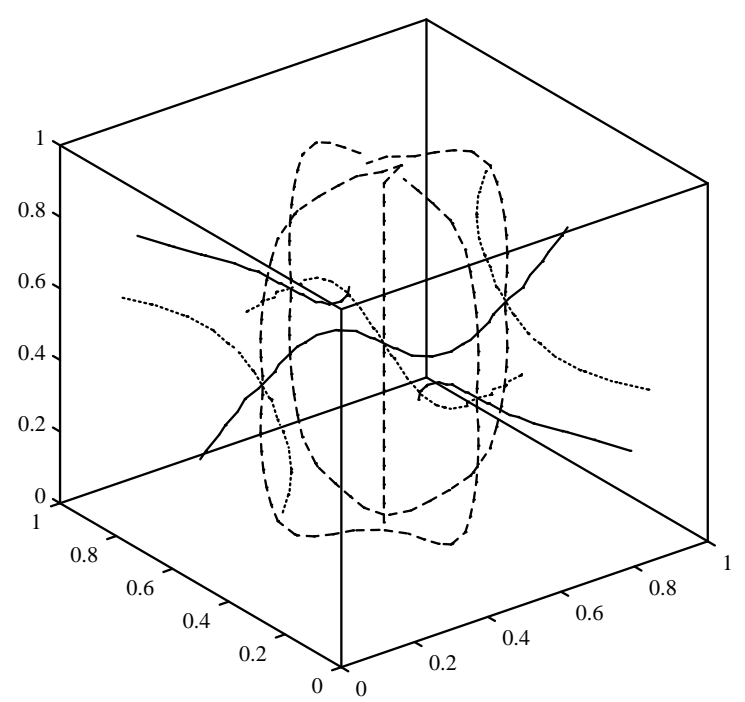

FIG. 13. Final map with five singularities, obtained with the relaxation or conjugate gradient methods.

\section{REFERENCES}

[1] H. Brezis, J.-M. Coron, And E. Lieb, Harmonic maps with defects, Comm. Math. Phys., 107 (1986), pp. 649-705.

[2] Y. Chen, The weak solutions to the evolution problem of harmonic maps, Math. Z., 201 (1989), pp. 69-74.

[3] R. Cohen, S.-Y. Lin, And M. Luskin, Relaxation and gradient methods for molecular orientation in liquid crystals, Comput. Phys. Comm., 53 (1989), pp. 455-465.

[4] R. Cohen, R. Hardt, D. Kinderlehrer, S.-Y. Lin, and M. Luskin, Minimum energy configurations for liquid crystals: Computational results, in Theory and Applications of Liquid Crystals, IMA Vol. 5, Springer-Verlag, New York, 1987, pp. 99-122.

[5] E. Dean, R. Glowinski, And C. H. Li, Applications of operator splitting methods to the numerical solution of nonlinear problems in continuum mechanics and physics, in Mathematics Applied to Science, Academic Press, New York, 1988, pp. 13-64.

[6] M. Fortin AND R. Glowinski, Méthodes de lagrangien augmenté, in Méthodes Mathématiques de l'Informatique, 9, Dunod, Paris, 1982.

[7] F. C. Frank, On the theory of liquid crystals, Discuss. Faraday Soc., 25 (1958), p. 19-28.

[8] R. Gulliver, Harmonic mappings, symmetry and computer graphics, Preprint CMA-R-52-88, School of Mathematics, University of Minnesota, 1988.

[9] R. Gulliver, Fiber evolution in the heat flow of harmonic maps, in Nematics: Mathematical and Physical Aspects, J.-M. Coron, J.-M. Ghidaglia, and F. Helein, eds., 1991, pp. 173-178.

[10] F.-H. LiN, A remark about the map x/ $|x|$, C. R. Acad. Sci. Paris, 305 (1987), pp. 529-531.

[11] S.-Y. LIN, Numerical analysis for liquid crystal problems, Ph.D. thesis, University of Minnesota, Minneapolis, 1987.

[12] S.-Y. Lin AND M. Luskin, Relaxation methods for liquid crystal problems, SIAM J. Numer. Anal., 26 (1989), pp. 1310-1326.

[13] C. W. Oseen, The theory of liquid crystals, Trans. Faraday Soc., 29 (1933), pp. 883-899.

[14] T. RIVIÈRE, Existence of infinitely many weakly harmonic maps into spheres for non constant boundary datas, Preprint 9419, Centre de Mathématiques et de Leurs Applications, Cachan, France, 1994.

[15] D. SERRE, private communication.

[16] P.-G. De Gennes And J. Prost, The Physics of Liquid Crystals, Clarendon Press, Oxford, 1993. 